\title{
Contrôle de constitutionnalité des lois de finances et protection des droits fondamentaux en République Démocratique du Congo
}

\author{
Par Marcel Wetsh'Okonda*
}

\section{Résumé}

La Constitution congolaise du 18 février 2006 contient un catalogue étoffé de droits fondamentaux, y compris sociaux. Pour assurer leur mise en œuvre, le Gouvernement et le Parlement devraient en tenir compte, le premier lors de l'élaboration des lois de finances annuelles et le second au moment de l'adoption de celles-ci. Tel ne semble pas, cependant, la situation sur terrain et ce, notamment à cause de la faiblesse du contrôle de constitutionnalité des lois de finances annuelles. C'est ce que la présente contribution s'efforce de montrer.

\begin{abstract}
The Congolese constitution of February 18, 2006, contains an extensive catalog of fundamental rights, including social rights. To ensure their implementation, the government and parliament should take them into account, the former when drafting the annual finance laws and the latter when adopting them. However, this does not seem to be the situation on the ground, mainly because of the weakness of the constitutional review of annual finance laws. This is what the present contribution seeks to show.
\end{abstract}

\section{Introduction}

Définies comme celles qui déterminent les prévisions des ressources et des charges de l'Etat de l'année ${ }^{1}$, les lois de finances annuelles, dont cellescommunément connues sous le nom de budget de l'Etat $^{2}$, présentent un intérêt capital, notamment en ce qu'elles permettent, au regard du volume des enveloppes allouées aux différentes rubriques qu'elles comportent, d'identifier les véritables priorités du Gouvernement.

* Par Marcel WETSH'OKONDA KOSO, Avocat au Barreau de Kinshasa/Gombe, Doctorant en droit public àl'Université de Kinshasa. E-mail : marcwetshk@yahoo.fr.

1 Articles 126, alinéas 1 et 3 et 175, alinéa 1 de la Constitution du 18 février 2006 telle que modifiée le 20 janvier 2011.

2 Aux termes de l'article 18 de la Loi n ${ }^{\circ} 11 / 011$ du 13 juillet 2011 relative aux finances publiques, les autres lois de finances sont les lois portant reddition de comptes, les lois de finances rectificatives et les lois portant ouverte des crédits provisoires. 
Sur la base de ce critère, il y a lieu de soutenir, preuve à l'appui, que, peu importe sa rhétorique, plus ou moins démagogique, celui-ci est plus préoccupé par les besoins sociaux de la population, la promotion du genre, la sécurité de l'Etat, les intérêts égoöstes de ses membres, etc. plutôt que par telle ou telle autre matière.

Comme toutes les autres, ces lois constituent essentiellement un produit de la conjugaison des efforts du Gouvernement et du Parlement, au premier revenant la prérogative exclusive de concevoir et d'initier les projets de lois de finances tandis que leur adoption est nécessairement le fait de l'autorité budgétaire qu'est le Parlement.

Ces deux institutions n'ont pas, cependant, les mains totalement libres en la matière. $\mathrm{Au}$ contraire, elles sont soumises à un certain nombre d'exigences, notamment d'ordre constitutionnel ${ }^{3}$.

Dès lors, la Cour constitutionnelle devrait s'assurer que lesdites exigences sont respectées et en tirer les conséquences qui s'imposent.

L'impératif de l'exercice de cette fonction juridictionnelle s'avère d'autant plus intéressant qu'au nombre de ces obligations constitutionnelles dont l'observation devrait être assurée, il convient de mentionner les droits fondamentaux, spécialement ceux économiques, sociaux et culturels.

L'objet de la présente contribution est justement de passer en revue les contraintes constitutionnelles en matière d'élaboration du budget de l'Etat avant de montrer combien la faiblesse, dans ce domaine particulier, du contrôle de constitutionnalité, impacte négativement la protection des droits fondamentaux.

\section{A. Les contraintes constitutionnelles en matière d'élaboration du budget}

De l'analyse de la Constitution, il se dégage que celle-ci comprend un certain nombre de dispositions en matière d'élaboration du budget dont les unes se rapportent à la forme tandis que les autres relèvent du fond.

\section{Les contraintes de forme}

Par contraintes de forme, il convient d'entendre celles qui s'attachent à la compétence et à la procédure applicable en matière budgétaire.

Comme précédemment indiqué, deux institutions ont qualité pour intervenir en matière d'élaboration des lois de finances annuelles : le Parlement et le Gouvernement, auxquels on peut ajouter le Président de la République.

3 Concrétisées et complétées par celles portées par la loi précitée sur les finances publiques (LOFIP) qui ne seront pas, cependant, évoquées dans la présente contribution. 
C'est ce qui se dégage principalement de l'article 126 de la Constitution aux termes duquel :

«L'Assemblée nationale et le Sénat votent les projets de loi de finances (...) Le projet de loi de finances de l'année (...) est déposé par le Gouvernement au Bureau de l'Assemblée nationale $(\ldots)^{4} »$.

La loi fondamentale détermine la procédure d'adoption des lois de finances annuelles, laquelle est ponctuée par six étapes principales. Primo : l'initiative des lois de finances annuelles revient exclusivement au Gouvernement à qui incombe l'obligation de déposer le projet de loi de finances annuelles et ce, au plus tard le quinze septembre de l'année 5 . Trois enseignements majeurs méritent d'être tirés à ce niveau de réflexion. D'abord, il n'existe pas de proposition de loi de finances annuelle (initiative parlementaire). Ensuite, pour être réguliers, les projets de loi de finances doivent être soumis non au Sénat mais à l'Assemblée nationale et à celle-ci seulement ${ }^{6}$. Enfin, il existe un délai butoir de soumission du projet de loi de finances de l'année à l'Assemblée nationale : le 15 septembre de chaque année. Qu'arrive-t-il lorsque ce délai n'a pas été respecté? Deux situations doivent être distinguées. D'une part, conformément à l'article 126, alinéa 6 de la Constitution, si le projet de loi n'a pas été déposé en temps utile pour que la loi subséquente soit promulguée avant le début de l'exercice budgétaire fixé au $1^{\mathrm{er}}$ janvier ${ }^{7}$, le Gouvernement peut solliciter l'ouverture des crédits provisoires auprès de l'Assemblée nationale et du Sénat. Au cas où ceux-ci ne se prononcent pas dans les quinze jours de cette demande, les dispositions du projet prévoyant ces crédits sont mises en vigueur par le Président de la République sur proposition du Gouvernement délibérée en conseil des ministres ${ }^{8}$. D'autre part, si le Gouvernement ne dépose pas le projet de loi de finances quinze jours avant la fin de la session parlementaire budgétaire du 15 septembre au 15 décembre$^{9}$, il est réputé démissionnaire ${ }^{10}$.

Secundo : à lire l'article 127 de la Constitution, le pouvoir d'amendement des projets de lois de finances de l'année reconnu aux parlementaires (députés et sénateurs) subit des limitations non négligeables.

Ils ne sont recevables que dans la mesure où ils tendent à la diminution des recettes ou à l'accroissement des recettes. La difficulté peut, cependant, être contournée s'ils sont assortis des propositions compensatoires.

4 Marcel WETSH'OKONDA KOSO, Les textes constitutionnels congolais annotés, Kinshasa, Editions de la Campagne pour les droits de l'homme au Congo, 2010, p. 476.

5 Article 130, alinéa 2 de la Constitution.

6 On notera, en passant, que les autres initiatives législatives sont déposées soit à l'Assemblée nationale soit au Sénat, les deux possibilités étant ouvertes à leurs auteurs.

7 Article 172 de la Constitution.

8 Article 126, alinéa 8 de la Constitution.

9 Article 115 de la Constitution.

10 Article 126, alinéa 7 de la Constitution. 
Tertio : la discussion des projets de loi de finances annuelles porte, devant l'Assemblée nationale, sur le texte déposé par le Gouvernement. En ce qui le concerne, le Sénat discute sur le texte voté, en première lecture, par l'Assemblée nationale ${ }^{11}$.

Quarto : à l'instar des lois organiques, les lois de finances annuelles sont votées à la majorité absolue des membres composant chaque chambre.

Quinto : en cas d'adoption de la loi en termes non identiques par les deux Chambres, une commission mixte paritaire est constituée. Faute, par celle-ci, de dégager un compromis sur la loi, la version adoptée par l'Assemblée nationale prévaut, à condition, toutefois, qu'elle soit adoptée par la majorité absolue des membres de celle-ci ${ }^{12}$.

Sexto : à moins de la renvoyer au Parlement pour une seconde délibération, le Président de la République promulgue la loi de finances de l'année dans un délai de quinze jours à dater de sa réception ${ }^{13}$. A défaut, elle entre en vigueur de plein droit ${ }^{14}$.

Comme on peut le constater, comparée à celle des autres $10 \mathrm{~s}^{15}$, la procédure d'élaboration des lois de finances de l'année recèle quelques spécificités ${ }^{16}$. Ce qui requiert une attention particulière de l'Assemblée nationale, du Sénat et du Gouvernement à qui incombent également un certain nombre d'obligations de fond.

\section{Les contraintes de fond}

La Constitution ne contient pas seulement des dispositions relatives à l'organisation et au fonctionnement des organes principaux de l'Etat ainsi qu'aux attributions de ceux-ci. Elle comprend aussi des dispositions plus substantielles liées aux droits fondamentaux. Celles-là

11 Article 132 de la Constitution.

12 Article 139 de la Constitution.

13 Article 137 de la Constitution.

14 Article 140 de la Constitution.

15 Pour aller plus loin sur le domaine de la loi en République démocratique du Congo, lire Marcel WETSH'OKONDA KOSO, « Domaines de la loi et du règlement et leur protection sous l'empire de la Constitution de la République démocratique du Congo du 18 février 2006 ", in Ivan MINGASHANG (dir.), La responsabilité du juriste face aux manifestations de la crise dans la société contemporaine. Un regard croisé autour de la pratique du droit par le Professeur Auguste Mampuya, Bruxelles, Editions Bruylant, 2018, pp. 771-807.

16 Sur le régime juridique des lois organiques, lire Marcel WETSH'OKONDA KOSO, « Un nouvel éclairage sur le régime juridique des lois organiques sous l'empire de la Constitution du 18 février 2006. A propos de l'arrêt de la Cour constitutionnelle n RConst. 212/216/2016 du 10 juin 2016 », Le Plumitif, Revue de droit congolais, $4^{\mathrm{ème}}$ année, $\mathrm{n}^{\circ} 4,2017$, pp. 11-36. En ce qui concerne les lois de programmation, lire Marcel WETSH'OKONDA KOSO, « Les lois de programmation sous l'empire de la Constitution du 18 février 2006 : inconstitutionnalité et constitutionnalité », in J. KAZADI MPIANA (dir.), La place de la justice constitutionnelle et administrative dans la consolidation de l'Etat de droit et la protection des droits fondamentaux. Mélanges en l'honneur du Professeur émérite Tshitambwe Kazadi Honoré (à paraître). 
font l'objet, selon la terminologie de Maurice Hauriou, de la Constitution politique tandis que celles-ci relèvent de la Constitution sociale ${ }^{17}$.

Ce sont les dispositions ressortissant à cette dernière qu'on qualifie des dispositions de fond. Il s'agit essentiellement de celles contenues essentiellement dans le deuxième titre de la loi fondamentale relatif aux droits humains, aux libertés fondamentales et aux devoirs du citoyen et de l'Etat allant des articles 11 à 67.

Ces dispositions constitutionnelles s'articulent autour de quatre chapitres consacrés respectivement aux droits civils et politiques (articles 11 à 33); aux droits économiques, sociaux et culturels (articles 34 à 49) et aux droits dits collectifs (articles 50 à 61) ${ }^{18}$.

L'adverbe essentiellement s'explique par le fait que toutes les dispositions en rapport avec les droits fondamentaux ne sont pas contenues dans le deuxième titre de la Constitution, d'autres étant disséminées çà et là dans les autres titres de celle-ci. Tel est le cas, entre autres, du droit à la nationalité (article 10) et de celui du peuple à la souveraineté permanente sur les ressources naturelles (articles 5 et 9) inclus dans le premier titre, sans oublier le droit à la libre administration des provinces (article 3). Il en va de même du droit à l'indépendance du pouvoir judiciaire consacré notamment par les articles 149 et 150 , contenus dans le troisième titre et 220 du septième ${ }^{19}$.

Selon une opinion largement répandue, la réalisation des droits économiques, sociaux et culturels requiert des prestations positives de la part de l'Etat. Ce qui leur a valu la qualification de droits de créance. Pour le dire plus clairement, ledit Etat doit mobiliser des moyens dont ceuxfinanciers appropriés pour l'exercice effectif de ces droits, notamment le droit à l'éducation, y compris dans sa composante de la gratuite de l'éducation fondamentale (articles 43 à 45). Il ne peut en être autrement lorsqu'on sait que, pour nous limiter à ce seul exemple, c'est le gouvernement et non les parents qui doivent prendre en charge les salaires des enseignants du secteur public. Dans le même ordre d'idées, des fonds appropriés devraient également être mobilisés, entre autres, pour construire des hôpitaux (article 47) et des logements sociaux (article 48), améliorer progressivement l'accès à

17 Maurice HAURIOU, Précis de droit constitutionnel, Paris, $2^{\text {ème }}$ édition, Dalloz, 2015. Sur cet élargissement de l'objet de la Constitution et le renouveau du droit constitutionnel qui en résulte, lire Frédéric JOEL AIVO, « Le juge et les droits fondamentaux. Retour sur un quart de siècle de jurisprudence (trop active) de la Cour constitutionnelle du Bénin », in Koffi AHADZI-NONOU, Dodzi KOKOROKO, Adama KPODAR et Frédéric JOEL AIVO (coord.), Démocratie en questions? Mélanges en l'honneur du professeur Théodore Holo, Toulouse, Presses de l'Université Toulouse 1 Capitole, 2017, pp. 445-486, surtout 448 et s.; Louis FAVOREU et alii, Droit consti-

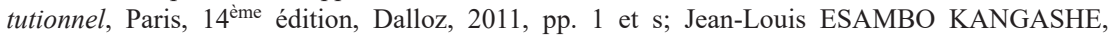
Le droit constitutionnel, Louvain-La-Neuve, Academia-L'Harmattan, 2013, pp. 7-8; Traité de droit constitutionnel congolais, Paris, L'Harmattan, coll. « Etudes africaines », 2017, pp. 19-22; Ambroise KAMUKUNY MUKINAY, Droit constitutionnel congolais, Kinshasa, Editions universitaires africaines, 2011, pp. 22-24.

18 Les devoirs du citoyen font l'objet des articles 62 à 67 de la Constitution.

19 Pour aller plus, loin, lire Marcel WETSH'OKONDA KOSO, Les perspectives des droits de l'homme dans la Constitution congolaise du 18 février 2006, Kinshasa, Editions de la Campagne pour les droits de l'homme au Congo, 2006. 
l'accès à l'eau potable et à l'électricité (article 48), la situation sociale des femmes (article 14) et des enfants (article 41), etc.

En réalité, les prestations positives de l'Etat s'avèrent également nécessaires pour la satisfaction des autres droits fondamentaux ${ }^{20}$. A titre illustratif, le droit d'électorat comme celui d'éligibilité ne relèvent pas des droits économiques, sociaux et culturels. Ils constituent plutôt des droits politiques. Leur mise en œuvre, à la faveur de l'organisation des élections régulières, n'en nécessite pas moins un budget conséquent. Le droit au juge et son corollaire, le droit à l'indépendance du pouvoir judiciaire indépendant en fournissent également une éloquente illustration. Leur effectivité passe nécessairement par l'adoption d'un budget à la hauteur des besoins en matière de construction des infrastructures judiciaires et pénitentiaires, d'entretien et d'équipement des cours et tribunaux et de rémunération des magistrats.

En définitive, comme l'article 60 de la Constitution l'affirme, le respect des droits de l'homme et des libertés fondamentales consacrés dans la Constitution s'impose aux pouvoirs publics et à toute personne. Le Gouvernement à qui revient l'initiative des lois de finances annuelles et le Parlement qui les adopte ne font pas exception à la règle. Encore faut-il, cependant, que leurs œuvres communes soient soumises à un contrôle de constitutionnalité efficace ${ }^{21}$.

\section{B. L'impact négatif de la faiblesse du contrôle de constitutionnalité des lois de finances annuelles sur la protection des droits fondamentaux}

Bien qu'il soit techniquement possible, le contrôle de constitutionnalité des lois de finances annuelles se heurte encore, dans les faits, à beaucoup d'obstacles. Ce qui impacte, négativement, la mise en œuvre des droits fondamentaux.

20 Lire à ce sujet, Jean-François AKANDJI KOMBE, « Droits économiques, sociaux et culturels », in Joël ANDRINTSIMBAZOVINA, Hélène GAUDIN, Jean-Pierre MARGUENAUD, Stéphane RIALS et Frédéric SUDRE (dir.), Dictionnaire des droits de l'homme, Paris, PUF, 2012, pp. 322-325; Philippe BLACHER, " Droits fondamentaux (classification)», in Dominique CHAGNOLLAUD et Guillaume DRAGO (dir.), Dictionnaire des droits fondamentaux, Paris, Dalloz, 2010, p. 280; Marie-Claire PONTHOREAU, Droit(s) constitutionnel(s) comparé(s), Paris, Economica, 2010, pp. 161-163; Danièle LOCHAK, Droits de l'homme, Paris, $3^{\mathrm{ème}}$ et $2^{\mathrm{ème}}$ édition, La Découverte, 2009 et 2005, pp. 38-43 et 41-43; Allan MCCHESNEY, Promouvoir et défendre les droits économiques, sociaux et culturels : un manuel, New York, American Association for Advancement et Science et Huridocs, 2002.

21 Sur les fondements et les modalités d'exercice de la justice constitutionnelle dans le monde en général et en République démocratique du Congo en particulier, lire avec intérêt Dieudonné KALUBA DIBWA, La justice constitutionnelle en République démocratique du Congo, LouvainLa-Neuve et Kinshasa, Academia-L'Harmattan-Academia-L'Harmattan, 2013. 


\section{La possibilité technique du contrôle}

La Constitution du 18 février 2006 érige la République démocratique du Congo en un Etat de droit. Dans un tel Etat, le droit se présente sous la forme d'une pyramide au sommet de laquelle trône la Constitution, les autres normes juridiques se partageant les échelons inférieurs. Sous peine de sanction par le juge, les normes inférieures sont soumises à celles supérieures, spécialement à la Constitution (Etat de droit formel). Celle-ci comportant un catalogue plus ou moins étoffé des droits fondamentaux, toutes les normes juridiques sont, ipso jure, soumises à ceux-ci (Etat de droit substantiel). L'Etat de droit est, ainsi, à la fois un Etat de justice et un Etat des libertés ${ }^{22}$.

Les articles 157 à 169 de la Constitution susvisée, complétée par la Loi organique portant organisation et fonctionnement de la Cour constitutionnelle sont consacrées aux attributions ainsi qu'à l'organisation et au fonctionnement de celle-ci.

Au nombre des attributions de cette juridiction, il convient de mentionner le contrôle de constitutionnalité des lois et des actes ayant force de $\operatorname{loi}^{23}$. Les lois de finances annuelles seraient-elles concernées par ce contrôle?

Le caractère général de la formulation des dispositions constitutionnelles pertinentes incline à répondre à cette interrogation par l'affirmative. Selon l'article 159, alinéa 1 de la Constitution, la Cour constitutionnelle est chargée du contrôle de constitutionnalité des lois et des actes ayant force de lois. Les lois de finances annuelles relevant de cette catégorie juridique, elles ne sauraient, logiquement, échapper au contrôle de constitutionnalité.

Encore faut-il qu'elles soient déférées devant la Cour. A ce sujet, deux scénarios sont possibles : le contrôle préventif ou a priori et le contrôle répressif ou a posteriori.

Dans le premier cas de figure, les actes juridiques concernés ne sont pas encore promulgués (les lois), mis en application (règlements intérieurs des chambres et des institutions d'appui à la démocratie) ou ratifiés (accords et traités internationaux). En cas d'arrêt d'inconstitutionnalité, ils resteront dans cet état.

L'initiative du contrôle de constitutionnalité est tantôt obligatoire tantôt facultatif. Il est obligatoire en ce qui concerne les lois organiques et les règlements intérieurs tant des chambres parlementaires que des institutions d'appui à la démocratie ${ }^{24}$. Il est déclenché par le président de la République pour celles-là et, selon le cas, les présidents des chambres parlementaires ou des institutions d'appui à la démocratie pour ceux- $\mathrm{ci}^{25}$.

Le contrôle est plutôt facultatif à l'égard des autres actes susceptibles de contrôle de constitutionnalité devant la Cour constitutionnelle, plus précisément les lois ordinaires.

22 Marcel WETSH'OKONDA KOSO, « La consolidation de l'Etat de droit à l'épreuve de l'indépendance des juges constitutionnels en République démocratique du Congo », Annuaire congolais de justice constitutionnelle, Volume 4, 2019, pp. 109-131.

23 Articles 139, 160 et 162 de la Constitution.

24 Articles 124 et 160, alinéa 2 de la Constitution.

25 Pour aller plus loin, lire la Loi organique $n^{\circ} 13 / 026$ du 15 octobre 2013 portant organisation et fonctionnement de la Cour constitutionnelle. 
Dans les quinze jours de leur transmission, celles-ci peuvent être soumises à la Cour constitutionnelle, pour les besoins de leur contrôle de constitutionnalité, par le président de la République ou le Premier ministre. Elles peuvent également l'être, dans un délai de quinze jours à dater de leur adoption, par le président de l'Assemblée nationale ou du Sénat, ou encore par un dixième de députés ou de sénateurs ${ }^{26}$.

La doctrine est divisée au sujet du régime juridique du contrôle de constitutionnalité des lois de finances ${ }^{27}$. Pour les uns, celles-ci sont des lois ordinaires. En tant que telles, elles sont susceptibles du contrôle facultatif de constitutionnalité. Tout en admettant que les lois de finances ressortissent des lois ordinaires, d'autres soutiennent qu'elles devraient plutôt faire l'objet du contrôle obligatoire de constitutionnalité. Cette position doctrinale trouve son fondement juridique dans les dispositions combinées des 126, alinéa 2 et 124 de la Constitution. Selon le premier, l'Assemblée nationale et le Sénat votent les projets de lois de finances dans les conditions prévues par la loi organique visée à l'article 124 de la Constitution.

En ce qui le concerne, ce dernier dispose, entre autres, que les lois organiques ne peuvent être promulguées qu'après déclaration par la Cour constitutionnelle obligatoirement saisie par le Président de la République, de leur conformité à la Constitution dans un délai de quinze jours ${ }^{28}$.

Les articles 160 et 161 de la Constitution forment le soubassement juridique du deuxième cas de figure, celui du contrôle répressif ou a posteriori de constitutionnalité. Comme son nom l'indique, celui-ci concerne les actes juridiques déjà entrés en vigueur, en l'occurrence les lois, les ordonnances-lois (actes ayant force de loi) et les actes règlementaires. Déclenché, par voie d'action ou au moyen d'une requête ou encore par voie d'exception devant n'importe quelle juridiction, il peut aboutir, en cas d'inconstitutionnalité, à un arrêt de nullité de plein droit des actes juridiques concernés ${ }^{29}$.

Point n'est besoin d'aller plus loin pour réaliser que, loin d'être une simple vue de l'esprit, le contrôle de constitutionnalité des lois de finances de l'année relève du possible. La compétence de la Cour constitutionnelle en la matière ne fait l'ombre d'aucun doute. Certes, il existe une controverse juridique sur les modalités pratiques exactes dudit contrôle, les uns évoquant le contrôle préventif ou a priori et obligatoire tandis que les autres soutiennent plutôt la thèse du contrôle facultatif. Le contrôle répressif ou a posteriori est également envisagé.

26 Article 139 de la même Constitution.

27 Lire Marcel WETSH'OKONDA KOSO, La protection des droits de l'homme par le juge constitutionnel congolais, Paris, L'Harmattan, Coll. « Notes de cours », 2016, pp. 73-74.

28 KITOKO KIMPELE, « Le contrôle par le juge constitutionnel des lois de finances ", in Recueil des messages, discours et instructions du Premier président de la Cour suprême de justice et président du Conseil supérieur de la magistrature (de 2011 à 2013), Kinshasa, Cour suprême de justice, 2013, pp. 63-64.

29 Article 168, alinéa 2 de la même Constitution. 
Faute de jurisprudence de la Cour constitutionnelle, en la matière, chacun continue de camper sur sa position. Pire encore, les exigences constitutionnelles de forme et de fond relatives aux lois de finances demeurent relativement formelles.

\section{L'impact négatif de l'ineffectivité du contrôle}

De prime abord, il importe de souligner que, dans certains Etats, le juge constitutionnel exerce une influence non négligeable dans la procédure d'élaboration des lois de finances annuelles, dont les budgets de l'Etat, en contraignant le Gouvernement et le Parlement à respecter scrupuleusement les exigences constitutionnelles en la matière. Le phénomène est connu sous le nom de la constitutionnalisation du droit des finances publiques ${ }^{30}$.

Parlant de l'expérience française en la matière, Louis Favoreu relève que :

"Chaque année, la loi de finances est déférée au juge constitutionnel par les parlementaires, voire par le Premier ministre ou le président de l'Assemblée nationale lui-même. Ces affaires, s'ajoutant à celles de l'article 40, constituent une véritable jurisprudence d'où il ressort que le droit budgétaire et même le droit fiscal sont tributaires du respect des dispositions constitutionnelles ou assimilées telles que les lois organiques et les règlements d'assemblé.

Certains ont même pu, à propos de la décision récente du 24 décembre 1979 annulant la loi de finances pour 1980, regretter que l'on verse dans un excès de juridisme. Ce qui montre que l'opinion, en France, n'est pas familiarisée avec le contrôle de constitutionnalité des lois ${ }^{31}$ ».

Une expérience similaire a été vécue par certains Etats d'Amérique latine. C'est du moins ce qui se dégage de la lecture de Carlos Bernal Pulido. Selon ce dernier auteur :

"Dans le cas de la Colombie, la Cour constitutionnelle a spécifié les conditions structurelles que les prisons doivent remplir pour garantir les droits des prisonniers (sentence SU-995 de 1999), elle a indiqué que le salaire des fonctionnaires publics ne peuvent pas être gelé, mais doit être augmenté chaque année en fonction de

30 Sur la Constitutionnalisation du droit pénal congolais, lirePaul-Gaspard NGONDANKOY NKOYea-LOONGYA, « Répression du Président de la République et du Premier ministre en droit congolais; esquisse de formation d'un droit constitutionnel pénal de fond ", in Jean-Paul SEGIHOBE BIGIRA et Ivon MINGASHANG (dir.), Le droit pénal entre douleur et enchantement dans le contexte contemporain. Liber amicorium Raphael Nyabirungu mwene Songa, Bruxelles, Bruylant, 2021, pp. 995-1062; Corneille WASENDA N'SONGO, « La constitutionnalisation du droit pénal congolais ou l'exigence du respect des dispositions constitutionnelles : difficile conciliation? ", Revue de droit africain, $\mathrm{n}^{\circ} 92,2019$, pp. 532-562; La constitutionnalisation du droit pénal congolais ou l'exigence du respect des dispositions constitutionnelles : difficile conciliation?, Thèse de doctorat en droit, Université de Paris 1, Panthéon Sorbonne, 2019.

31 Louis FAVOREU, «L'apport du Conseil constitutionnel au droit public », in La Constitution et son juge, Paris, Economica, 2014, p. 1107. 
l'inflation (sentences C-1433 2000, C-1064 de 2001, de C-1017 de 2003 et C-931 de 2004), et elle a déclaré inconstitutionnelles des normes instaurant un système de financement de logement qu'elle considérait incommode et contraire au droit au logement décent (sentences C-038, C-700, C-747 et C-995 de 1999), elle établit que le gouvernement ne pouvait remettre en cause les conditions salariales et les prestations sociales dans des conventions collectives au profit des employés du secteur public (sentences C-038 et 754 de 2004), elle a protégé le droit des vendeurs ambulants de travailler de façon informelle dans la rue (sentence T-772 de 2003), et elle a estimé insuffisante la protection que le gouvernement a donnée aux populations déplacées du fait d'actes de violence (sentence T-025 de 2004) ${ }^{32} »$.

A en croire Antonis Panagopoulos, une situation similaire a prévalu en Grèce où, au nom du principe constitutionnel d' égalité devant la loi, la Cour suprême faisant office de juge constitutionnel n'a pas hésité de juger que les rémunérations des magistrats doivent être supérieures à celles des fonctionnaires et égales à celles des parlementaires ${ }^{33}$. Ce qui ne pouvait que conforter l'indépendance du pouvoir judicaire.

Plus intéressant encore est le cas de l'Allemagne où, dans le silence de la Cour constitutionnelle de 1949 sur les droits sociaux, la Cour constitutionnelle n'en a pas moins assuré la protection de ceux-ci en se fondant sur le caractère social de l'Etat consacré par la même Constitution $^{34}$.

Le moins qu'on puisse dire est qu'aussi paradoxal que cela puisse paraître, il n'en va pas encore ainsi en République démocratique du Congo où plusieurs critiques sont formulées au sujet des budgets de l'Etat alors même que, jusque-là-ceci expliquant peut-être cela-aucun d'entre eux n'a été sanctionné par la Cour constitutionnelle.

Deux exemples suffiront pour nous en convaincre. En premier lieu, au sujet des droits sociaux des fonctionnaires et agents publics de l'Etat, Buabwa Wa Kayembe note qu' :

" En dépenses, la Commission focalise son attention sur le budget d'investissement, ne se souciant pas parfois des dépenses non sincères et souvent sous-évaluées de

32 C. BERNAL PULIDO, "Droits fondamentaux, juristocratie et hyper présidentialisme en Amérique latine ", in A. MARTIN (dir.), Le glaive et la balance. Droits de l'homme, justice constitutionnelle et démocratie en Amérique latine, Paris, L'Harmattan, 2012, p. 36.

33 Antonis PANAGOPOULOS, Modèle américain ou européen de justice constitutionnelle? Etude comparative à travers le cas hellénique, thèse pour le doctorat en droit, Université Aix-Marseille III, 2011, pp. 76-77.

34 Marie-Claire PONTHOREAU, Droit(s) constitutionnel(s) comparé(s), Paris, Economica, p. 359. Sur le contrôle de constitutionnalité des lois de finances au Gabon, lire S. KWAHOU, « Le contrôle de la constitutionnalité des lois de finances au Gabon », Revue de droit africain, $13^{\text {ème }}$ année, $\mathrm{n}^{\circ}$ 50, 2009, pp. 228-252. En ce qui concerne l'expérience du Bénin, lire Nicaise MEDE, « Le juge constitutionnel, un juge financier? », in Oumarou NAREY (dir.), La justice constitutionnelle, Paris, L'Harmattan, 2016, pp. 245-261; Moussa ZAKI, " Le contrôle de constitutionnalité des lois de finances : serpent de mer ou miroir aux alouettes?, in Oumarou NAREY (dir.), La justice constitutionnelle, op.cit., pp. 263-290. 
certains ministères qui sont néanmoins à la base en phase d'exécution de grands dérapages dans le domaine des finances publiques du pays.

Les projets de budget en RDC sont très rarement porteurs d'espoir en matière de social ou du vécu quotidien de la population. Rien de concret n'y est prévu dans le domaine du logement, de la santé, de l'enseignement, d'eau potable, d'électricité, etc. ${ }^{35} \gg$.

Curieusement, alors que cela est techniquement possible, à notre connaissance, aucune initiative n'a été prise pour saisir la Cour constitutionnelle en inconstitutionnalité de ces budgets peu respectueux des droits fondamentaux, du caractère social de l'Etat consacré expressément dès l'article premier de la loi fondamentale et des droits sociaux sus évoqués.

Faudrait-il, pour cela, jeter la pierre aux justiciables qui pècheraient par leur silence complice face à la méconnaissance de leurs propres droits?

Le second exemple appelle à plus de prudence. Tirant les leçons des tentatives infructueuses de décentralisation du passé dont celles initiées par le professeur Vunduawe et l'honorable Malumba Mbangula, le constituant a levé l'option en faveur de la décentralisation politique. Désormais, non seulement celle-ci est consacrée par la loi fondamentale mais, mieux encore, le constituant a pris le soin de déterminer lui-même les attributions des provinces ${ }^{36}$ tout en les dotant des ressources humaines propres ${ }^{37}$. C'est dans cette perspective que s'inscrit l'article 175 de la Constitution qui mérite d'être cité in extenso :

"Le budget des recettes et des dépenses de l'Etat, à savoir celui du pouvoir central et des provinces, est arrêté chaque année par une loi.

La part des recettes à caractère national allouée aux provinces et établie à $40 \%$. Elle est retenue à la source.

La loi fixe la nomenclature des autres recettes locales et la modalité de leur répartition ».

Il va sans dire que, mises en application, ces dispositions constitutionnelles sont de nature à faciliter la réussite de la nouvelle décentralisation ${ }^{38}$.

35 Mathias BUABUA WA KAYEMBE, Les finances publiques congolaises. Pouvoir central, provinces, entités territoriales décentralisées, Kinshasa, Editions universitaires africaines, 2013, p. 137.

36 Articles 201 à 206 de la Constitution.

37 Articles 195 à 198 de la même Constitution. Pour aller plus loin sur les finances publiques des provinciales et entités locales, lire Georges NDJOLI BOMPE, La décentralisation fiscale en République démocratique du Congo; Evolution du système, comparaison et impact sur le développement, Kinshasa, Editions Droit et Société (DES), 2018; SHOTSHA KATSHUNGA, Finances locales, Kinshasa, Editions KATSH, 2017.

38 Pour aller plus loin lire Jean-Michel KUMBU ki NGIMBI (dir.), La décentralisation territoriale en République démocratique du Congo sous le régime de la Constitution du 18 février 2006 : bilan et perspectives, Kinshasa, Editions de la Campagne pour les droits de 1'homme au Congo, 2014. 
Tel n'était pas encore le cas il y a une dizaine d'années. C'est dans ces conditions que, constatant que la loi budgétaire annuelle de l'Etat pour l'exercice 2008 n'avait pas répondu correctement aux exigences de ces énoncés constitutionnels, deux sénateurs, Vincent de Paul Lunda Bululu ${ }^{39}$ et Jacques Djoli Eseng'Ekeli40 avaient pris l'initiative de déposer chacun une requête en inconstitutionnalité devant la défunte Cour suprême de justice exerçant alors, à titre provisoire, les attributions dévolues à Cour constitutionnelle. A notre connaissance, ces recours sont demeurés sans suite.

On retiendra que, faute de contrôle efficace de constitutionnalité, les exigences constitutionnelles en matière d'élaboration des lois de finances annuelles ne sont pas encore suffisamment prises en compte en République démocratique du Congo. La réalisation des droits fondamentaux, singulièrement ceux sociaux en subit le contrecoup. Deux facteurs majeurs semblent à la base de cet état de choses.

D'abord, au lieu de déférer ces lois à la Cour constitutionnelle pour l'examen de leur conformité à la Constitution, le commun de congolais se contente de dénoncer leur caractère peu respectueux de leurs droits fondamentaux. Ensuite-cela expliquant peut-être ceci-les quelques fois qu'elle a été appelée à se prononcer sur la conformité à la Constitution des lois de finances annuelles, la Haute cour a choisi de s'emmurer dans le silence. Ce déni de justice suffit-il aux citoyens au sens vrai du terme pour croiser les bras devant n'importe quelle loi de finances annuelle? La question reste posée.

\section{Bibliographie}

\section{Textes juridiques}

Loi organique $\mathrm{n}^{\circ} 13 / 026$ du 15 octobre 2013 portant organisation et fonctionnement de la Cour constitutionnelle, Journal officiel de la République démocratique du Congo, $54^{\mathrm{ème}}$ année, $\mathrm{n}{ }^{\circ}$ spécial, 18 octobre 2013.

Loi $\mathrm{n}^{\circ} 11 / 011$ du 13 juillet 2011 relative aux finances publiques, Journal officiel de la République démocratique du Congo, 60 ${ }^{\mathrm{ème}}$ année, $\mathrm{n}^{\circ}$ spécial, 7 octobre 2019, pp. 185-248.

39 Lire la requête inscrite au numéro RConst.068/TSR en ligne à l'adresse http://www.afrimap.o rg/english/images/documents/ Requete\%20Lunda\%20Bululu\%20CSJ.pdf (10 décembre 2009); voir aussi http://www.lepotentiel.com/afficher_article.php?id_edition=\&id_article=59700 (10 décembre 2009).

40 Lire la requête inscrite au numéro RConst.068/TSR en ligne à l'adresse http://www.afrimap.o rg/english/images/documents/ Requete\%20Lunda\%20Bululu\%20CSJ.pdf (10 décembre 2009); voir aussi http://www.lepotentiel.com/afficher_article.php?id_edition=\&id_article=59700 (10 décembre 2009). 


\section{Ouvrages}

Frédéric JOEL AIVO, « Le juge et les droits fondamentaux. Retour sur un quart de siècle de jurisprudence (trop active) de la Cour constitutionnelle du Bénin », in Koffi AHADZI-NONOU, Dodzi KOKOROKO, Adama KPODAR et Frédéric JOEL AIVO (coord.), Démocratie en questions? Mélanges en l'honneur du professeur Théodore Holo, Toulouse, Presses de l'Université Toulouse 1 Capitole, 2017.

Jean-François AKANDJI KOMBE, "Droits économiques, sociaux et culturels ", in Joël ANDRINTSIMBAZOVINA, Hélène GAUDIN, Jean-Pierre MARGUENAUD, Stéphane RIALS et Frédéric SUDRE (dir.), Dictionnaire des droits de l'homme, Paris, PUF, 2012, pp. 322-325.

Philippe BLACHER, "Droits fondamentaux (classification) », in Dominique CHAGNOLLAUD et Guillaume DRAGO (dir.), Dictionnaire des droits fondamentaux, Paris, Dalloz, 2010, p. 280.

C. BERNAL PULIDO, « Droits fondamentaux, juristocratie et hyper présidentialisme en Amérique latine ", in A. MARTIN (dir.), Le glaive et la balance. Droits de l'homme, justice constitutionnelle et démocratie en Amérique latine, Paris, L'Harmattan, 2012.

Mathias BUABUA WA KAYEMBE, Les finances publiques congolaises. Pouvoir central, provinces, entités territoriales décentralisées, Kinshasa, Editions universitaires africaines, 2013.

Jean-Louis ESAMBO KANGASHE, Traité de droit constitutionnel congolais, Paris, L'Harmattan, coll. «Etudes africaines », 2017.

Jean-Louis ESAMBO KANGASHE, Le droit constitutionnel, Louvain-La-Neuve, Academia-L'Harmattan, 2013.

Louis FAVOREU et alii, Droit constitutionnel, Paris, 14 ${ }^{\text {ème }}$ édition, Dalloz, 2011.

Maurice HAURIOU, Précis de droit constitutionnel, Paris, $2^{\mathrm{ème}}$ édition, Dalloz, 2015.

Dieudonné KALUBA DIBWA, La justice constitutionnelle en République démocratique du Congo, Louvain-La-Neuve et Kinshasa, Academia-L'Harmattan-Academia-L'Harmattan, 2013.

Ambroise KAMUKUNY MUKINAY, Droit constitutionnel congolais, Kinshasa, Editions universitaires africaines, 2011

Jean-Michel KUMBU ki NGIMBI (dir.), La décentralisation territoriale en République démocratique du Congo sous le régime de la Constitution du 18 février 2006 : bilan et perspectives, Kinshasa, Editions de la Campagne pour les droits de l'homme au Congo, 2014.

Danièle LOCHAK, Droits de l'homme, Paris, $3^{\text {ème }}$ et $2^{\text {ème }}$ édition, La Découverte, 2009 et 2005.

Nicaise MEDE, « Le juge constitutionnel, un juge financier? », in Oumarou NAREY (dir.), La justice constitutionnelle, Paris, L'Harmattan, 2016, pp. 245-261.

Allan MCCHESNEY, Promouvoir et défendre les droits économiques, sociaux et culturels : un manuel, New York, American Association for Advancement et Science et Huridocs, 2002.

Georges NDJOLI BOMPE, La décentralisation fiscale en République démocratique du Congo; Evolution du système, comparaison et impact sur le développement, Kinshasa, Editions Droit et Société (DES), 2018. 
Paul-Gaspard NGONDANKOY NKOY-ea-LOONGYA, " Répression du Président de la République et du Premier ministre en droit congolais; esquisse de formation d'un droit constitutionnel pénal de fond », in Jean-Paul SEGIHOBE BIGIRA et Ivon MINGASHANG (dir.), Le droit pénal entre douleur et enchantement dans le contexte contemporain. Liber amicorium Raphael Nyabirungu mwene Songa, Bruxelles, Bruylant, 2021, pp. 995-1062.

Marie-Claire PONTHOREAU, Droit(s) constitutionnel(s) comparé(s), Paris, Economica, 2010.

SHOTSHA KATSHUNGA, Finances locales, Kinshasa, Editions KATSH, 2017.

Marcel WETSH'OKONDA KOSO, « Les lois de programmation sous l'empire de la Constitution du 18 février 2006 : inconstitutionnalité et constitutionnalité ", in J. KAZADI MPIANA (dir.), $L a$ place de la justice constitutionnelle et administrative dans la consolidation de l'Etat de droit et la protection des droits fondamentaux. Mélanges en l'honneur du Professeur émérite Tshitambwe Kazadi Honoré (à paraître).

Marcel WETSH'OKONDA KOSO, « Domaines de la loi et du règlement et leur protection sous l'empire de la Constitution de la République démocratique du Congo du 18 février 2006 », in I. MINGASHANG (dir.), La responsabilité du juriste face aux manifestations de la crise dans la société contemporaine. Un regard croisé autour de la pratique du droit par le Professeur Auguste Mampuya, Bruxelles, Editions Bruylant, 2018, pp. 771-807.

Marcel WETSH'OKONDA KOSO, La protection des droits de l'homme par le juge constitutionnel congolais, Paris, L'Harmattan, Coll. « Notes de cours », 2016.

Marcel WETSH'OKONDA KOSO, Les textes constitutionnels congolais annotés, Kinshasa, Editions de la Campagne pour les droits de l'homme au Congo, 2010.

Marcel WETSH'OKONDA KOSO, Les perspectives des droits de l'homme dans la Constitution congolaise du 18 février 2006, Kinshasa, Editions de la Campagne pour les droits de l'homme au Congo, 2006.

Moussa ZAKI, «Le contrôle de constitutionnalité des lois de finances : serpent de mer ou miroir aux alouettes?, in Oumarou NAREY (dir.), La justice constitutionnelle, Paris, L'Harmattan, 2016, pp. 263-290.

\section{Articles}

Louis FAVOREU, «L'apport du Conseil constitutionnel au droit public », in La Constitution et son juge, Paris, Economica, 2014.

S. KWAHOU, « Le contrôle de la constitutionnalité des lois de finances au Gabon », Revue de droit africain, $13^{\text {ème }}$ année, n 50,2009 , pp. 228-252.

Corneille WASENDA N'SONGO, « La constitutionnalisation du droit pénal congolais ou l'exigence du respect des dispositions constitutionnelles : difficile conciliation? », Revue de droit africain, $\mathrm{n}^{\circ} 92,2019$, pp. 532-562.

Marcel WETSH'OKONDA KOSO, « La consolidation de l'Etat de droit à l'épreuve de l'indépendance des juges constitutionnels en République démocratique du Congo », Annuaire congolais de justice constitutionnelle, Volume 4, 2019, pp. 109-131. 
Marcel WETSH'OKONDA KOSO, « Un nouvel éclairage sur le régime juridique des lois organiques sous l'empire de la Constitution du 18 février 2006. A propos de l'arrêt de la Cour constitutionnelle ${ }^{\circ}$ RConst. 212/216/2016 du 10 juin 2016 ", Le Plumitif, Revue de droit congolais, $4^{\mathrm{ème}}$ année, $\mathrm{n}^{\circ} 4,2017$, pp. 11-36.

\section{Autres documents}

KITOKO KIMPELE, " Le contrôle par le juge constitutionnel des lois de finances ", in Recueil des messages, discours et instructions du Premier président de la Cour suprême de justice et président du Conseil supérieur de la magistrature (de 2011 à 2013), Kinshasa, Cour suprême de justice, 2013.

Antonis PANAGOPOULOS, Modèle américain ou européen de justice constitutionnelle? Etude comparative à travers le cas hellénique, thèse pour le doctorat en droit, Université Aix-Marseille III, 2011.

Requête inscrite au numéro RConst.068/TSR en ligne à l'adresse http://www.afrimap.org/english/imag es/documents/ Requete\%20Lunda\%20Bululu\%20CSJ.pdf (10 décembre 2009); voir aussi http://w ww.lepotentiel.com/afficher_article.php?id_edition=\&id_article=59700 (10 décembre 2009).

Requête enregistrée au numéro RConst.068/TSR en ligne à l'adresse http://www.afrimap.org/english /images/documents/Requete\%20Lunda\%20Bululu\%20CSJ.pdf (10 décembre 2009); voir aussi http://www.lepotentiel.com/afficher_article.php?id_edition=\&id_article=59700 (10 décembre 2009).

Corneille WASENDA N'SONGO, La constitutionnalisation du droit pénal congolais ou l'exigence du respect des dispositions constitutionnelles : difficile conciliation?, Thèse de doctorat en droit, Université de Paris 1, Panthéon Sorbonne, 2019. 